\title{
Algorithms, approximation, and empirical studies in behavioral and computational social choice-Preface
}

\author{
Judy Goldsmith · Jörg Rothe
}

Published online: 12 September 2013

(C) Springer Science+Business Media Dordrecht 2013

This special issue is devoted to behavioral social choice and computational social choice, two rapidly emerging, interdisciplinary fields at the interface of, on the one hand, social choice theory (which traditionally belongs to economics and political science) and, on the other hand, behavioral sciences and computer science. The main focus of the papers collected here is on algorithms and approximation as well as on empirical and experimental studies, and both original research papers and survey papers are included.

Preliminary versions of many of the papers in this special issue were presented in the Special Session on Computational Social Choice at the 12th International Symposium on Artificial Intelligence and Mathematics (ISAIM 2012), which took place in Fort Lauderdale, USA, in January 2012. However, this special issue is not a comprehensive proceedings of the ISAIM 2012 computational social choice special session, but rather consists of a selection of the best contributions presented at the conference, plus some carefully selected papers that fit the special issue theme. All papers accepted for publication here have run through the regular reviewing process of The Annals of Mathematics and Artificial Intelligence, with at least two reviewers for each submission and up to three rounds of reviews per paper. The reviews have been particularly detailed and helpful, and the editors are very grateful to all the reviewers for their time and invaluable effort to improve the submissions.

The topics covered in this special issue range from the contributions that behavioral social choice can make to computational social choice (in particular, when the observed data is highly incomplete), to the approximability of social welfare optimization in multiagent resource allocation, to judgement aggregation from the

J. Goldsmith $(\bowtie)$

Department of Computer Science, University of Kentucky, Lexington, KY 40506, USA

e-mail: goldsmit@cs.uky.edu

J. Rothe

Institut für Informatik, Heinrich-Heine-Universität Düsseldorf, 40225 Düsseldorf, Germany

e-mail: rothe@cs.uni-duesseldorf.de 
perspective of approximation, to algorithmic aspects of manipulation, bribery, and control in elections.

- A Behavioral Perspective on Social Choice, by Popova, Regenwetter, and Mattei, uses the Netflix competition data (many people's ratings of subsets of a very large set of movies) to illustrate the pitfalls of statistical extrapolation from sparse preference data. As they say, "The supreme goal of behavioral social choice is to investigate social choice procedures empirically while avoiding unnecessary and/or unsubstantiated assumptions about human behavior."

- Developing the Aggregate Empirical Side of Computational Social Choice, by Plassmann and Tideman, argues that, based on the election data that is currently extant, the spatial model of vote casting is a reasonable statistical model of human voting.

- A Survey of Approximability and Inapproximability Results for Social Welfare Optimization in Multiagent Resource Allocation, by Nguyen, Roos, and Rothe, starts from the observation that "social welfare optimization is a computationally intractable task in many settings." This paper pulls together results about approximability of optimization problems with bundle utilities and k-additive utilities with respect to utilitarian, egalitarian, and Nash product social welfare.

- Approximately Classic Judgement Aggregation, by Ilan Nehama, looks at the problem of learning the truth, rather than a preferred outcome, from many agents. Nehama relaxes the two main constraints assumed in classical judgement aggregation mechanisms, consistency and independence, and instead considers mechanisms that only approximately satisfy these constraints.

- Bribery in Voting with CP-Nets, by Mattei, Pini, Rossi, and Venable, investigates the computational complexity of changing the outcome of elections, under a variety of voting rules, by means of bribing voters to change their votes, when votes are given in terms of CP-nets.

- Challenges to Complexity Shields That Are Supposed to Protect Elections Against Manipulation and Control: A Survey, by Rothe and Schend, starts from the observation that many manipulation and control problems in voting, despite their NP-hardness, might be hard to solve only on subsets of the problem instances that may not arise frequently in practice. They look instead at ways to challenge these NP-hardness results, including typical-case and experimental analyses, approximability, and fixed-parameter complexity as more practical measures of the hardness of manipulating and controlling elections, also under a variety of voting rules and specific types of manipulation and control.

We thank all the authors for their wonderful contributions to this special issue, and wish the readers to take much pleasure in reading them.

Judy Goldsmith and Jörg Rothe

August, 2013 